\title{
CHAPTER 98
}

RECTANGULAR RESONATORS FOR HARBOUR ENTRANCES

by

William James*

\section{ABSTRACT}

A complete theoretical analysis of the behaviour of resonators built into the breakwaters of a harbour entrance would be extremely difficult. Th1s paper describes an approximate development for extreme values of resonator geometry and shows how a combination of these cases explains certain observed phenomena for intermediate shapes.

The paper also describes comprehensive laboratory tests on resonators and draws attention to certain significant discrepancies from present design practice. In particular, the paper indicates the extent to which optimum resonator geometry depends upon the harbour entrance width and demonstrates that such resonators can be designed to prevent any penetration of waves into harbours, without restricting shipping.

\section{INTRODUCTION}

A resonator is taken to be a short rectangular branch canal, completely closed on three sides, built orthogonally on to the entrance breakwaters of a harbour (see Fig.1). Only the action of such resonators in parallel straight breakwaters has been investigated and this geometry is identical to that studied by Valembois in 1953 (ref.1). In this present paper the effect of distributed reflections from the harbour is not considered; this study pertains only to the geometry of such resonators and their action on waves entering harbours.

The dimensions of the resonator should be optimized such that a mass of water contaned within the resonator and that part of the entrance channe1 contiguous with the resonator mouth (here termed the "junction element" as shown in Fig.1), has a resonance frequency equal to that of the harmful incident wave that should be totally reflected back into the ocean. For the purposes of this paper the lowest frequency eigenvalue is used; for batteries of resonators tuned to cover a wide frequency band, resonance will also occur at higher harmonics.

Limiting the treatment to semı-infinite oceans and harbours results in simpler mathematics but introduces complications in the experiments, since the wave generator channel and harbour must be completely decoupled. Further simplifications in the mathematical treatment result from the use of first order linear wave theory and the assumption of irrotational motion. Such development w111 accord closely with the tsunami-type waves 11 kely to excite range action in real harbours but experimental waves are necessarily Stokian and, for accuracy, non-linear effects must be accounted for. Consequently, in this study, wave measurement was effected by micrometers and corrections were applied to render the readings correct to the nearest 0.005 inch.

* Lecturer, University of Natal. 


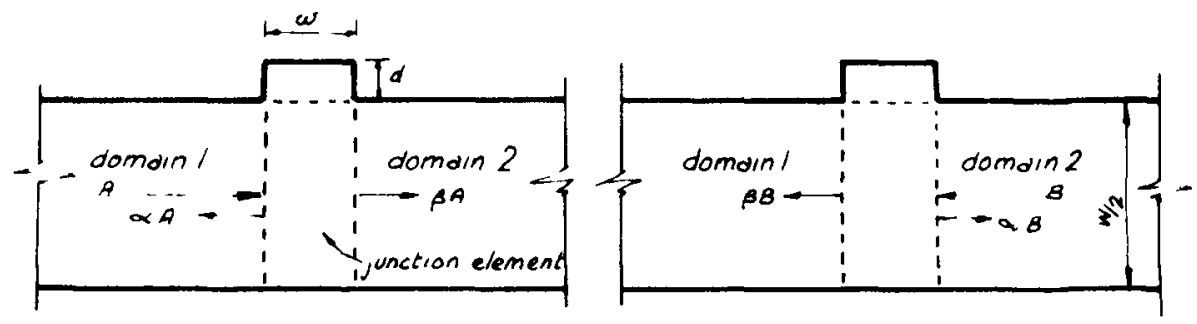

Fig.1 Wave components

\section{MATHEMATICAL TREATMENT}

Evidently the zone of protection provided by a resonator in the downstream direction, at resonance, is limited to a width of one half the incident wavelength (ref.2). Hence wave energy transmitted into the downstream domain will increase directly with increase in channel width above one wavelength, for the case where resonators are placed on both sides of the entrance channel. It follows that a resonator investigation of this type may be limited to one-dimensional propagation. Note that, though the assumption of plane waves is invalid near the junction element, two-dimensional motion becomes one-dimensional at distances of the order of twice the depth from the discontinuity, for these channel widths. Moreover, researchers have found that this limitation is not at all serious, provided that no transverse resonance occurs (ref.3). Transverse resonance is likely to occur when the basin width is close to an integral number of half-wavelengths .

The resonator geometry may be intermediate in shape between extremes that are either long and narrow, when the motion 18 in a direction normal to that of the waves in the entrance channel, or short and wide, when the motion is parallel to that of the entrance channel.

These cases are denoted mode I and mode II respectively (see Fig.2). It is assumed that the complicated motion for intermediate geometries can be approximated by superposition of these unidirectional components. Each mode is considered in turn and then a combination is shown to accord with observations for an intermediate case. The effect of turbulent dissipation is also discussed.

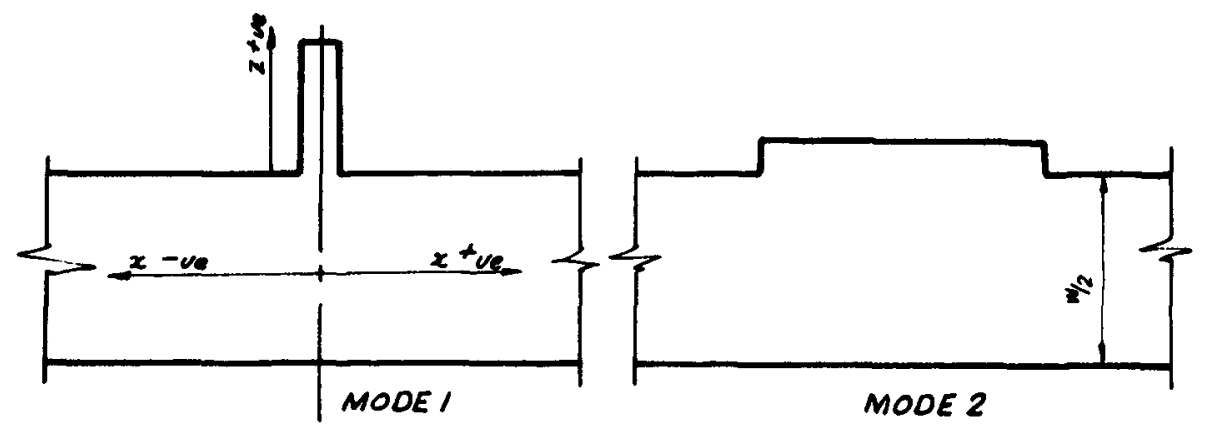

Fig. 2 Extreme cases. 
Mode I

Notation is defined at the end of this paper.

For mode I,

$$
w W<\lambda^{2}
$$

Hence: (a) the water surface of the junction element remains approximately horizontal and (b) the origins for the resonators and the channel may be assumed to coincide.

Using complex representation, the reflected wave is written:

$$
\eta=\bar{\alpha} a e^{j(k x+o t)}
$$

The water surface for the original incldent wave, the reflected wave, the partial clapotis in the resonators and the transmitted wave may be matched for each contiguity:

$$
\bar{\imath}+\bar{\alpha}=\bar{\beta}=(1+\bar{R}) \bar{\theta}
$$

The equation of continuity for the junction element is:

or:

$$
\begin{aligned}
\sum_{i, 2, R}\left\{\int_{h}^{0}\left(-\frac{\partial \phi}{\partial x}\right)_{x=0} d y\right\}=w W \frac{\partial \eta}{\partial t} \\
k \omega W \bar{\beta}=-j\{W(\bar{i}-\bar{\alpha}-\bar{\beta})-2 w \bar{\theta}(1-\bar{R})\}
\end{aligned}
$$

for equal depths throughout.

Equations (1) and (2) can be solved for $|\alpha|,|\beta|,|\theta|, \hat{\alpha}, \hat{\beta}, \hat{\theta}$ in terms of $|R|, \hat{R}$, and, in this study, an E1liot 803 computer was used to tabulate the results. All results were checked against the expression for the conservation of energy developed below.

\section{Mode II}

In this case the $\bar{\theta}$ and $\bar{\theta} \bar{R}$ waves are parallel to the main channel. The waves are matched for discharge and surface continuity again at each channel discontinuity as follows: $\bar{i}+\bar{\alpha}=\bar{\theta}(1+\bar{R})$

$$
\begin{aligned}
& \bar{\beta}=\bar{\theta}\left(1+\bar{R} e^{2 j k \omega}\right) \\
& W(\bar{l}-\bar{\alpha})=\bar{\theta}(1-\bar{R})(W+2 d) \\
& W \beta=\bar{\theta}(W+2 d)\left(1-\bar{R} e^{2 j k \omega}\right)
\end{aligned}
$$

These equations were solved, checked against energy conservation and tabulated using an I.B.M. 1620 computer.

\section{Energy conservation}

The energy of the reflected and transmitted waves is equal to that of the original incident wave less the energy dissipated in the resonators and junction element.

Proceeding from $E=\frac{1}{2} \rho \iint g \eta^{2} d x d z+\frac{1}{2} \rho \iint\left(\phi \frac{\partial \phi}{\partial y}\right)_{y=0} d x d z$

we get, for no dissipation, $|\alpha|^{2}+|\beta|^{2}=1$

For tsunamis and the experimental waves, viscous attenuation is negligible in the resonators. However, turbulence at the upstream edge is considerable and a lumped head loss can be postulated at this point:

$$
H_{L}=a(|\theta|-|\theta||R|)=K \frac{u|u|}{2 g}
$$


Equation (3) then leads to:

$$
|\alpha|^{2}+|\beta|^{2}+|\theta|^{2}\left(1-|R|^{2}\right) \frac{2 \omega}{W}=1
$$

This allows $H_{L}$ to be computed from observations of $|\alpha|,|\beta|$ and $|\theta|$.

$\mathrm{U}$ should include allowances for:

(a) local high velocities at the upstream re-entrant,

(b) the overall flow pattern in the junction element,

(c) the vertical velocity distribution.

The problem can be simplified by considering a steady-state flow pattern applicable to resonant conditions only and 1 gnoring the local effects of surface deformations. Thus the transverse velocity profile along an equipotential emanating from the upstream edge of the resonator provides a good basis. Extreme velocities close to the edge can be reduced to manageable proportıons by ignoring velocities within a nominal boundary layer and the vertical velocity distribution can be assumed to be the same as that given by Airy theory under a node in a standing wave. Using Schwarzian transforms for the upper half-plane the co-ordinates for given incremental values of $\psi$ can be computed along the equipotential starting from the sharp edge. The velocity $q$ is given by these differences and $K$ can be calculated from

$$
\int_{-h}^{0} \int_{0}^{l} \bar{q}|\bar{q}| d s d y=\frac{1}{k} 2 g a(|\theta|-|\theta||R|)
$$

Intermediate geometry.

Any general combination of the type

$$
(\alpha, \beta)_{\text {total }}=(\alpha, \beta)_{I_{1}} f_{1}(w, d)+(\alpha, \beta)_{I I} f_{2}(w, d)
$$

where the subscripts refer to the results obtained from computations for modes $I$ and $I I$ and $f_{i}(w, d)$ denotes a simple function of the type $d /(w+d)$ and $w /(w+d)$ respectively, would reveal that:

(a) $\beta_{\text {minimum }} \neq 0$ and $\alpha_{\max } \neq 1$ genera1ly.

(b) for large $w$, there is an increasing tendency for the resonant value of $\mathrm{d} / \lambda$ to exceed a value of 0.25 .

(c) for large $w$, there is a second type of resonance.

(d) resonant values of $d / \lambda$ depend upon both $w$ and $w$.

\section{PRELIMINARY VERIFICATION.}

A suitable experimental programme could not be devised without preliminary pilot studies. The results of these tests are now described and refer to an isolated single resonator.

Apparatus. The narrow "perspex" flume and timber housing for opposed adjustable rectangular resonators is ahow in $\mathrm{fig} .3$.

The rear wall of each resonator cantilevered vertically downwards from a system of clamps, which traversed along both side walls for the full length of the resonator. Hence any variation of resonator width, which was effected by means of a false (upstream) side wall, necessitated a change in width of the rear wall. This was achieved by means of twin thin overlapping galvanised iron sheet 1 ips. An intermediate perspex wall was used to reduce the width of the entrance channel. For such tests filters were arranged at the upstream sharp edge of the 
intermediate wall and on the seaward side an efficient wave absorber was built.

Where the theoretical domain is semi-infinite, it is axiomatic that wave re-flections off the paddle are NOT allowed and wave filters were used for such decoupling devices. These filters were not effective at low wave steepnesses. Nevertheless, results were frequently obtained under stop/start conditions, i.e. before the initial waves would re 5 reflect and hence were of some value.

The desideratum that domain 2 be semi-infinite caused difficulty, because of insufficient space (in which to absorb all the energy of the transmitted wave.) Of necessity the writer adopted an absorber similar to that developed by Hamill (ref.4).

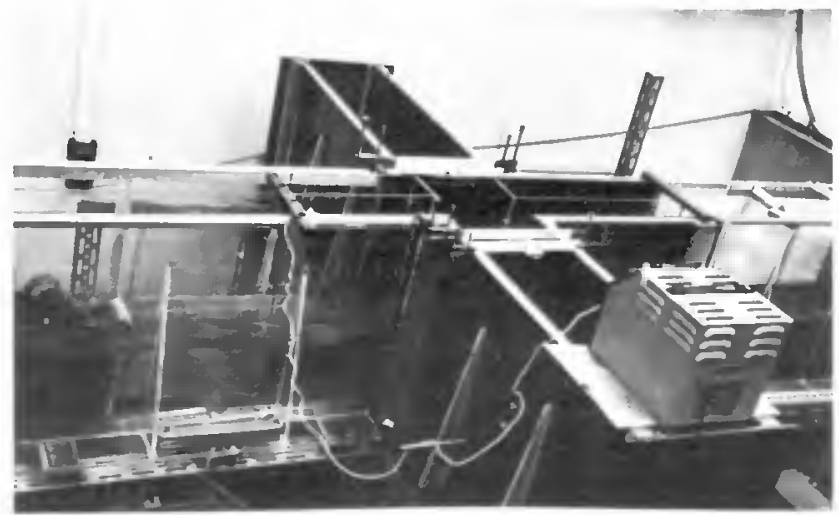

Fig.3 Preliminary apparatus.

Procedure. Envelope heights were directly measured to 0.01 " by means of a simple steel pointed probe of $0.10^{\prime \prime}$ diameter, detected with the aid of a standard CRO, the time base adjusted to give a stationary signal. The actual reading recorded was such that only 5 signals were obtained in any 10 consecutive waves.

Envelope heights were measured for each value of ' $d$ ', the length of the resonator. ' $\mathrm{d}$ ' was varied up to 8 times per value of resonator width, which was varied up to 7 times for each value of width of entrance channel. In all, four different entrance channel widths were tested. The wavelength was generally held constant. Wave periods were measured by timing 100 consecutive waves and wavelengths were then calculated by substitution into the first-order equations.

Every experimental "run" commenced from stil1 water conditions and measurements were conducted as soon as the partial clapotis in each domain had stabilised. Wave periods were measured both at the start and the end of the run.

\section{Results.}

Al1 experimental readings were plotted. Nodes and loops were measured off these plots and the coefficients of reflection and transmission 
were computed. In this pilot study no allowance was made for attenuation, non-linear effects, or experimental error.

The coefficients were then all plotted against the dimensionless tuning parameter $d / \lambda$ together with relevant results from theory (see Fig.4).

A number of photographs were taken of the entrance conditions at the resonator.

Discuss 1on.

The photographs indicated extreme turbulence throughout the resonator and junction element. Velocities of this nature will occur very seldom indeed in any ful1-scale structure but the photographs provided an obvious warning of the navigational hazards resulting from an irrational design.

An obvious result is the discrepancy of the resonating values of the tuning parameter from that prediced by theory, not only for the fundamental mode but also for higher harmonics. Simllar experimental results have in fact been obtained in analogous systems and have led to the formulation of "end corrections." The results of tests on resonators with curved entrances revealed further reductions in both the tuning parameter and the distance of the domain 1 partial clapotis from the resonator at resonance. This, of course, was to be expected.

\section{FURTHER EXPERIMENTS}

\section{Introduction.}

The pilot study provided reasonable agreement with the theoretical development, with the reservation that the "end correction" for the tuning parameter be evaluated empirically. However, the results obtalned for large resonator widths were inconclusive and it was apparent that the main objectives in the final programme should necessarily include:

(a) a substantial reduction of non-11near effects,

(b) improved measurement technique, with regard to both accuracy and speed,

(c) vastly improved decoupling devices,

(d) a study of the effect of entrance channel width,

(e) a study of the effect of large resonator widths.

Perhaps the most important object of this second programe was to check whether a single resonator behaved as a single-degree-of-freedom oscrllator, or, if not, to delineate the conditions under which this postulate did hold. Another important aim was to ascertain the validity of the linear theory used.

Apparatus. Experiments were carried out in the narrow flume, shown in Fig.5. It was hoped to shorten the upstream domann considerably in order to provide sufficient space for efficient measurement and absorbtion in the downstream domain. Eventually, however, a compromise was reached by the construction of the bend seen in Fig. 5 . 


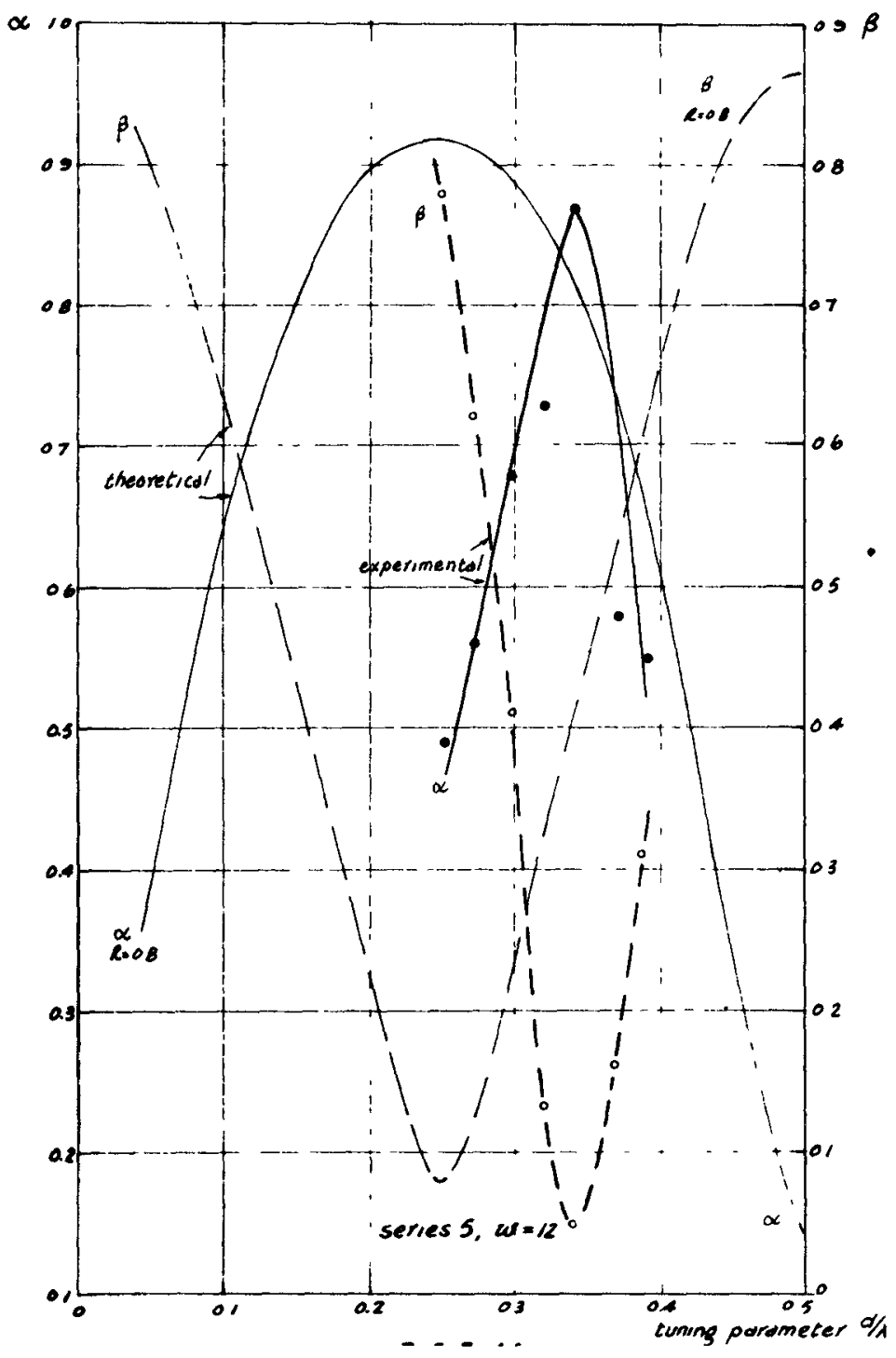

Fig. 4. Typical Preliminary Results. 
Transverse oscillations were avoided by prohibiting such troublesome frequencies. No low frequency vibrations greater than $0.0001^{\prime \prime}$ were detected. Nevertheless a special instrument was attached, in case parasitic oscillations developed from other sources (see p.i0).

Despite the smoothness of the perspex walls, side effects were easily visible under suitable low-level lighting. Disturbances radiated from the loops of the partial clapotis. Other sources of similar pseudorandom surface undulations were the filters, inconsistencies along the boundaries, vibrations inherent in the generator mechanism, and external vibrations, especially due to laboratory pumps and turbines and passing vehicular traffic. Surface tension was generally minimal, because of dispersive agents used in the measuring procedure, and consequently the "noise" resulting from meniscus inversion was probably of the order of 0.001 " in amplitude.

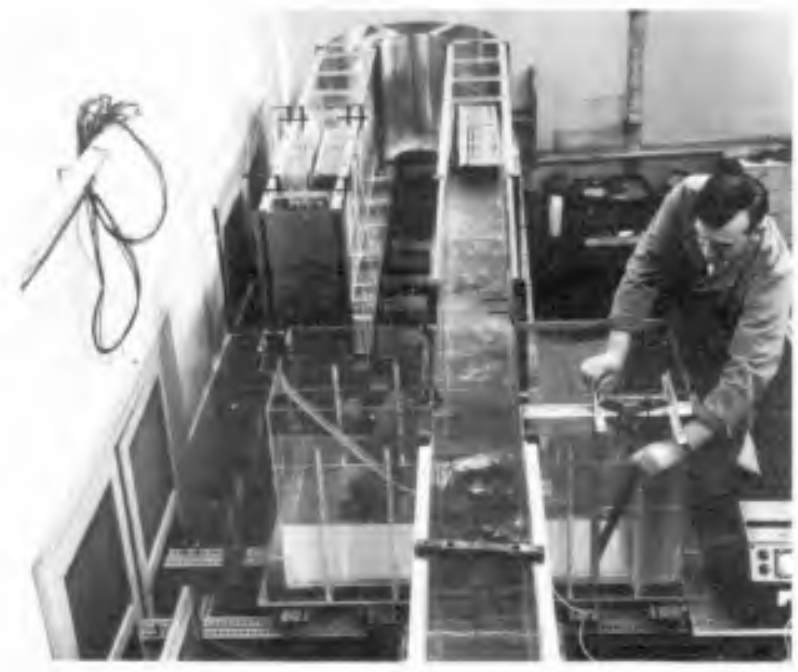

Fig. 5 Final Apparatus.

The incident wave periods and still water depths were chosen such that consistent waves were delivered at the resonators. For $\mathrm{T}<0.45$ secs. waves became too unstable and it was found that the standard deviation of wave heights varied inversely with still water depth. For reasonably shallow depths, this instability rendered wave measurement exceedingly difficult. Shallow still water depths also necessitated long probes which increased measuring errors and consequently the smallest depth tested was $5^{\prime \prime}$.

Standard deviations were measured by means of a "dekatron" counting unit and the probes described below. The count recorded the "number of crests greater than" (or "number of troughs less than") for each micrometer setting. Such a count was taken for batches of 100 consecutive waves at setting increments of $0.0005^{\prime \prime}$ throughout the range. 
Histograms were plotted from the differences in the counts for sequential settings, of which a sample is given in Fig.6. and these revealed symmetrical relative-frequency distributions. The computed standard deviations were of the order of $0.0015^{\prime \prime}$ for $a=0.25^{\prime \prime}$ and under the conditions obtaining. The coefficient of variation was less than $1 \%$. This compares very favourably with standard deviations quoted by other workers.

The symmetrical bell-shape shown in Fig. 6. greatly facilitated measurement; the mean reading is given by approximately half the number of a fairly large sample of waves, say 20 .

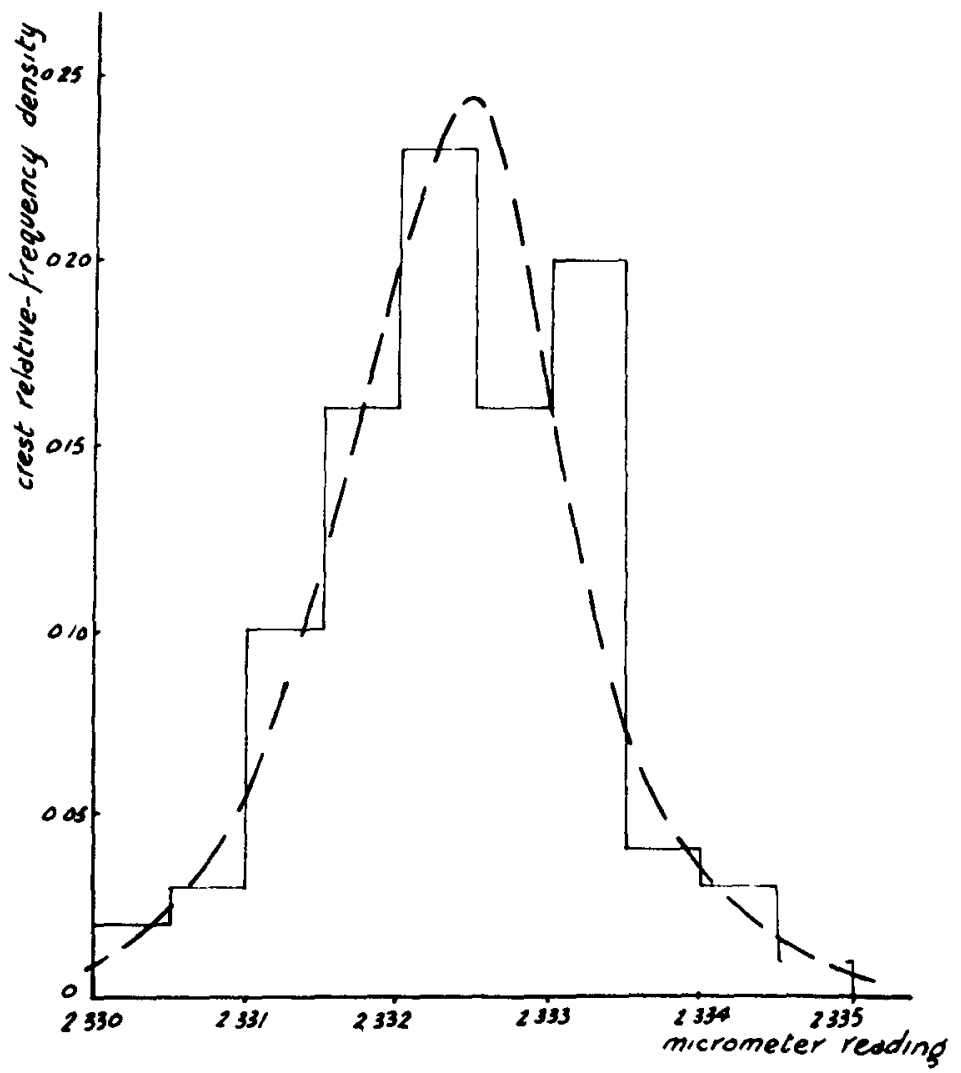

Fig. 6. Wave measurement histogram.

The writer did not investigate the reflection/transmission characteristics of the bend alone; the overall effect of the absorber downstream, the filters at each end of the bend, as well as the bend itself was examined. The filters were constructed of perforated zinc plate, 16 gauge (similar to those shown in Fig.7), $36^{\text {" }}$ long, at $0.5^{\prime \prime}$ pitch, extending throughout the depth of water and measured coefficients of transmissjon were of the order of $0.4-0.6$, depending upon wave length. 
At leâst two filters were arranged in each domain, whence the total "decoupling coefficient" for each domain was at worst 0.06 , assuming total reflection off the paddle and absorber.

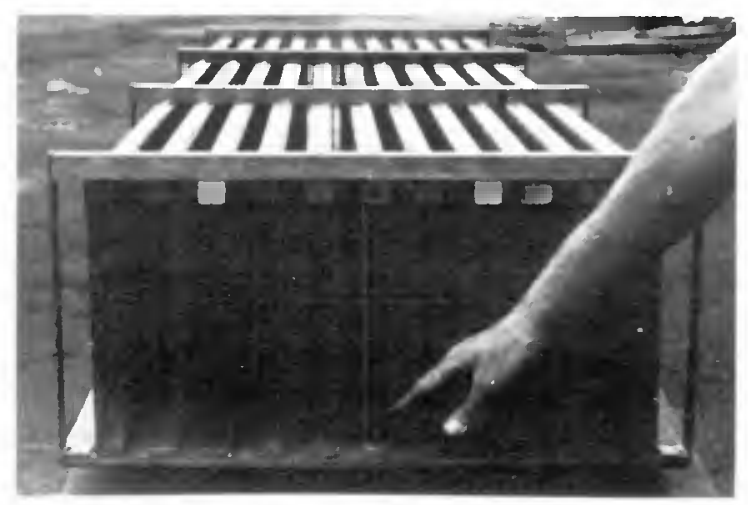

Fig. 7. Wave filters.

The reflectivity of the absorber used in the pilot study was found to be about 0.1 . Although this is considerably better than rubblemound absorbers - for which the value would have been about 0.3 , a desirable value would be $\alpha \neq 0.02$. The requirements of linear theory dictate that wave steepnesses be kept minimal and this in turn demands a highly efficient absorber.

Constructed in perspex, the final absorber was 6' $0^{\prime \prime} \times 9^{\prime \prime}$ and the perforations were $1^{\prime \prime}$ dia. at $2^{\prime \prime}$ centres. When fixed in position downstream of the bend, the overall gradient was less than 0.007 . The reflectivity of the entire domsin 2 set-up was measured and the readings adjusted for experimental errors and non-1inearity. The results demonstrated two points:

(a) decreasing transmission (from domain 1 to domain 2) with decreasing wavelength, due entirely to the dissipative forces.

(b) increasing reflectivity with increasing wavelength.

In an effort to detect long period fluctuations in water level, a 31 . separating flask was attached to the end of the flume. A column of water was positioned in a short horizontal section of capillary tube attached to a large diameter rubber bung and the lower end of the flask was connected to the flume through large diameter glass tubing. A change of water level in both the flume and the flask of $0.001^{1 "}$ would therefore displace the column of water several inches. The indicator had to be replaced regularly, as evaporation in the main flume caused sufficient reduction in water level. Short period fluctuations were ineffective because of the overall impedance of the arrangement. Within limits, the impedance could be adjusted by stopcocks. The instrument was attached to the flume behind the absorber, downstream of the bend. 
Major sources of error are surface tension effects. When these effects are reduced, e.g. by using very fine probes and limiting instantaneous contact to a depression of the crest surface rather than actually puncturing it, it is usually impossible to see any meniscus effect. Probes were fashioned out of fine stainless steel sewing needles, nominal diameter about $0.015 "$. The needles were coated with a thin layer of a P.V.C. akraline non-wetting paint, to ensure that no colum of water drained down off the probe as it emerged from a crest. Probably because of galvanic corrosion, it was necessary to maintain the sharp points by careful abrasion.

Probes were attached to micrometer heads, capable of being read to $0.0001 "$. The micrometer heads were securely mounted on heavy crossbars, clamped to the flume and the signal relayed to the cathode ray tube. A newer improved instrument uses a dial gauge, as shown in Fig.8. Another possible source of error is the sum of

(a) the depth to which the crest is actually depressed before a signal is obtained

(b) the height to which the surface of the crest is raised before contact is broken.

A dispersive agent was added continuously to reduce this and numerous very careful measurements were made on still water. The maximum correction was found to be $0.007^{\prime \prime}$. The extent of dynamic effects was not es tablished.

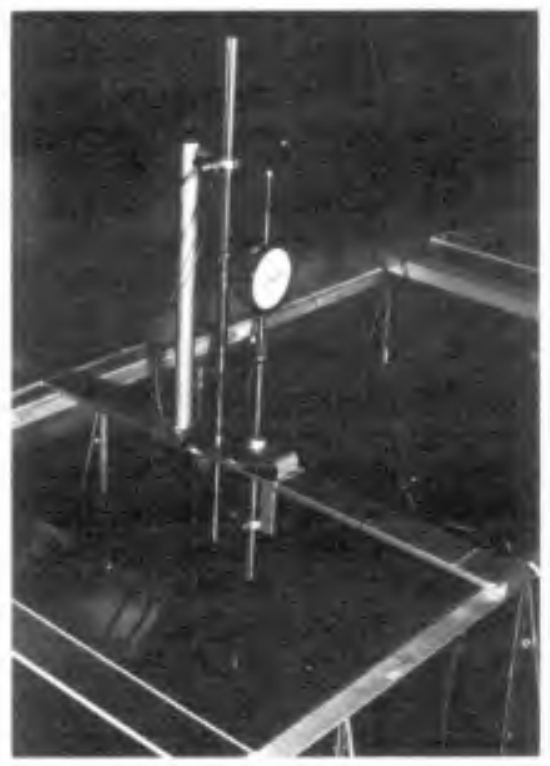

Fig. 8. Wave measurement. 
General Procedure.

Wave periods (for at least 100 consecutive waves) were measured both at the outset and at the conclusion of the experiment. Both transverse oscillations and seiches were successfully avolded, so that the generator was stopped only at the conclusion of each experimental "run" when the resonator geometry was adjusted. Experiments were controlled in the laboratory by simultaneous curve-plotting. In this way the parameters were chosen to yield the family of curves plotted in Fig.13.

Usual methods for evaluating the two primary components of the partial clapotis in both domains have two basic assumptions in common:

1. The fundamental waves have harmonic wave-form.

2. Only two such constituents are present.

Consequently, disregarding measuring errors, the accuracy of both methods increases with decrease in both amplitude and wavelength of the incident wave (because of reflectivity). It was found that values of $\propto$ calculated by the 3-point method were usually unreliable and the method was soon discarded in favour of the loop-and-node method. This enabled on-the-spot plotting of characteristics and thus greater flexibility and control of each experiment, a most important consideration.

On the other hand accurate scanning demands between 8 and 20 measurements of envelope height for each experiment, a somewhat protracted and tedious labour.

Data Processing. Resonator antinodes and loops and nodes for both domains were punched-up and a program was then written to correct for the measuring errors described above and to compute, inter alia, $\alpha$ and $\beta$.

These results were plotted as shown in Figs. 9 and 10 .

The effect of attenuation and non-1inearity is 1mmediate1y apparent, froin the fact that the plots do not tend to 1.0 or 0 , as the case may be, as $\mathrm{d} / \lambda \rightarrow 0$.

Discussion.

In assessing the accuracy of the results, the errors have been evaluated as follows:

(a) Systematic errors, due to attenuation and non-1inearity. These errors do not affect the derivation of the geometry for resonance but are of paramount importance in establishing absolute values of reflectivity and transmissivity. Corrections for both these errors can be applied easily:

(i) Attenuation. All values of $\alpha$ and $\beta$ should be corrected by multiplication by the reciprocal of $\beta_{0}$, the transmissivity at $d / \lambda=0$.

This operation yields even larger values of $\alpha_{0}$. The effect of domain 2 reflectivity at resonance is very $\operatorname{sma} 11$, $i . e$. usually $<0.01 \mathrm{~A}$ in amplitude in domain 1 . It is incorporated in the random errors discussed below.

(ii) Non-linearity. The analysis of real partial clapotis produces low values of $\alpha$, and negligible effect on $\beta$, when $\alpha$ is not very small. Corrections for this can be applied easily. 


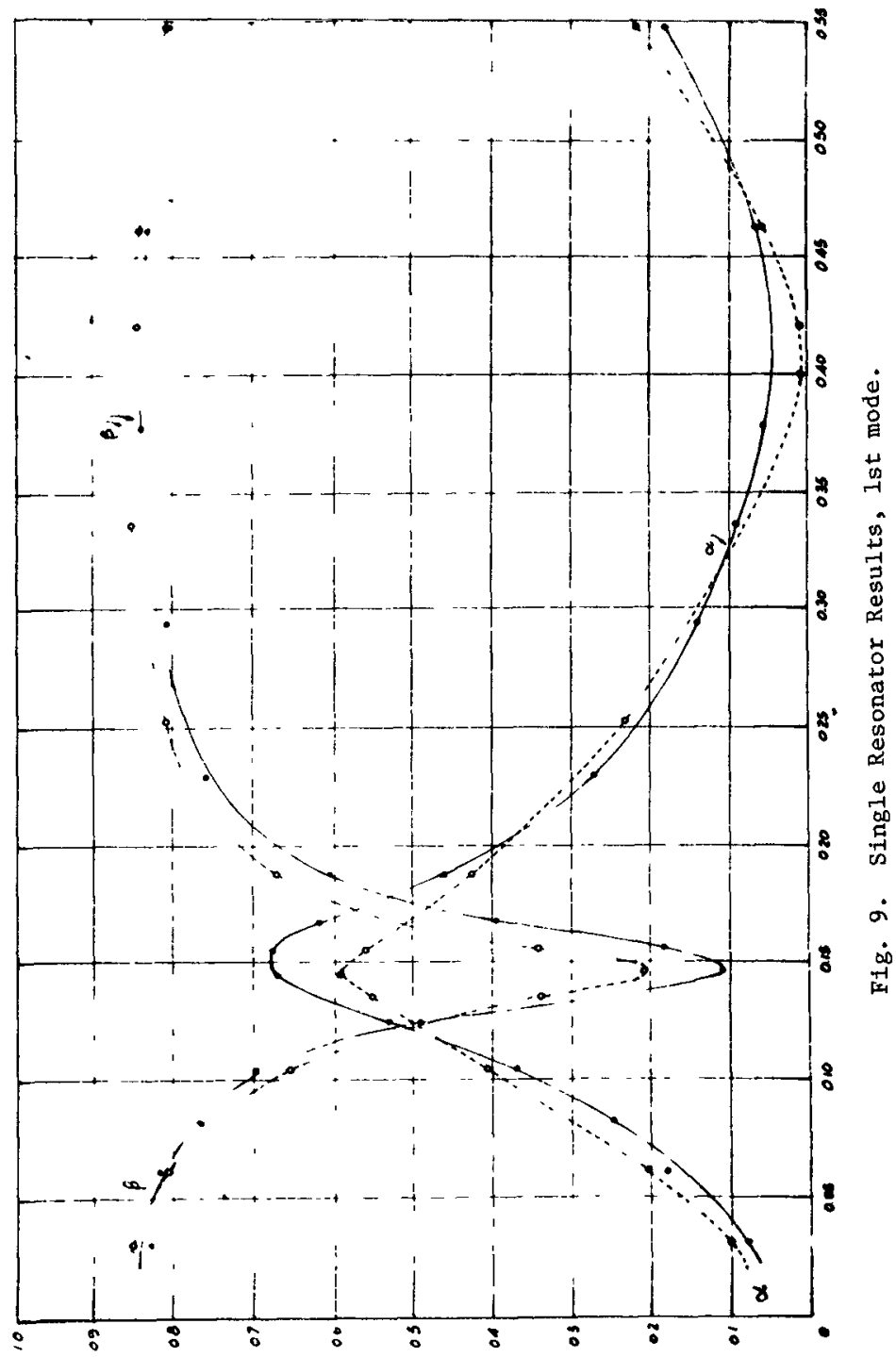




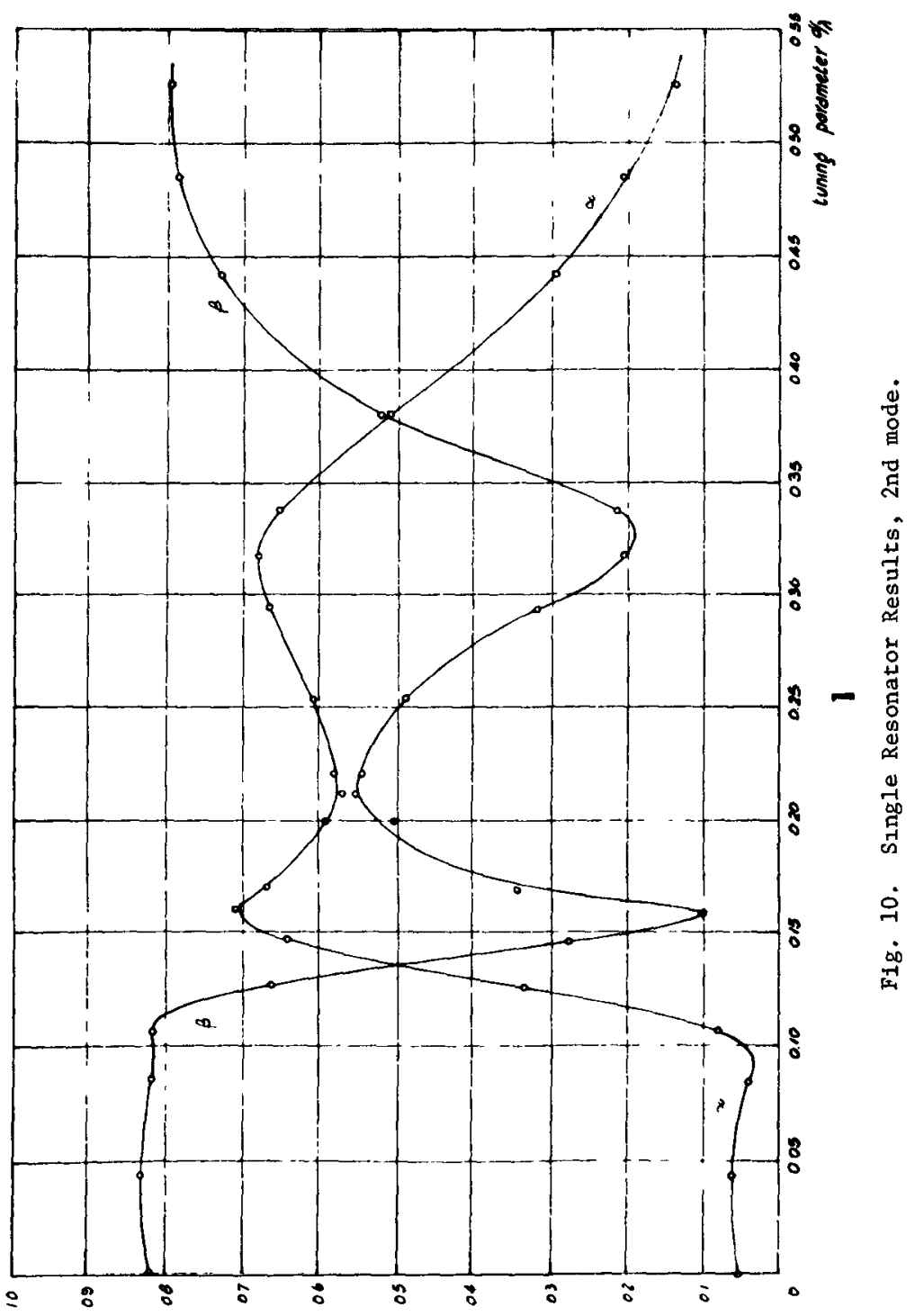


(b) Pseudo-random errors, attributable to generator instability, surface "noise", negative waves from domann 2 and the errors in the processes from measurement to plotting.

The writer did not attempt to assess individually such imponderable errors, as these are best calculated from the scatter obtaining in the graphs.

In error analyses, only graphs with a relatively high density of experimental results are admissible. As little scatter was found during the laboratory control of the experiments, the number of experiments was reduced to a minimum and, ipso facto, most of the curves plotted are unsuitable for error evaluation. However, certain curves were analysed and the probable error on the plot having the largest scatter of all the tests performed in the final programme was found to be 0.0146 . The writer therefore submits that the random error was not much more than $1 \%$.

The parameters of resonator geometry were rounded off to the second decimal place. The calculation of wavelength to this order of accuracy required an accuracy of measurement of wave perlod of 0.001 secs. and of still water depth of $0.05^{\prime \prime}$.

Figures 9 and 10, clearly indicate that the combined resonator and junction element acts as a two-degrees-of-freedom oscillator, under certain conditions. An appraisal of the $\alpha$ and $\beta$ curves reveals a gradual transition from the single-degree system to the two-degrees system and an eventual preponderance of the second mode of resonance as $w / \lambda$ increases beyond a value of 0.5 . Further appraisal reveals that this "critical" $w / \lambda$ increases with 1ncrease in $W / \lambda$.

The modes of oscillation are illustrated in figs. 11 and 12 respectively.

Note that the water surface sketched 18 exaggerated in the vertical scale; amplitudes were small and consequently the nodal 1 ines sketched are inaccurate. Nonetheless, the sketches elucidate the mechanisms at resonance and illustrate the transition between these states.

Results for the first resonant mode were finally combined in Fig.13.

\section{GENERAL CONCLUSIONS .}

For the sake of simplicity, only the points of primary importance are enumerated below:

1. Resonators can be applied in harbour engineering for the elimination of waves causing short period agitation, influx of sea sediments and/ or range action.

2. The results corroborate the results of the analytic theory developed and verify that the response of a short rectangular branch canal to periodical plane gravity waves propagated across the mouth is analogous to that of a single degree of freedom oscullator within certain limits:

For small values of $\mathrm{W} / \lambda$ and when $w / \lambda>0.5$, the resonator becomes a two degrees of freedom oscillator. For larger values of $W / \lambda$ this limiting value of $w / \lambda$ increases. 


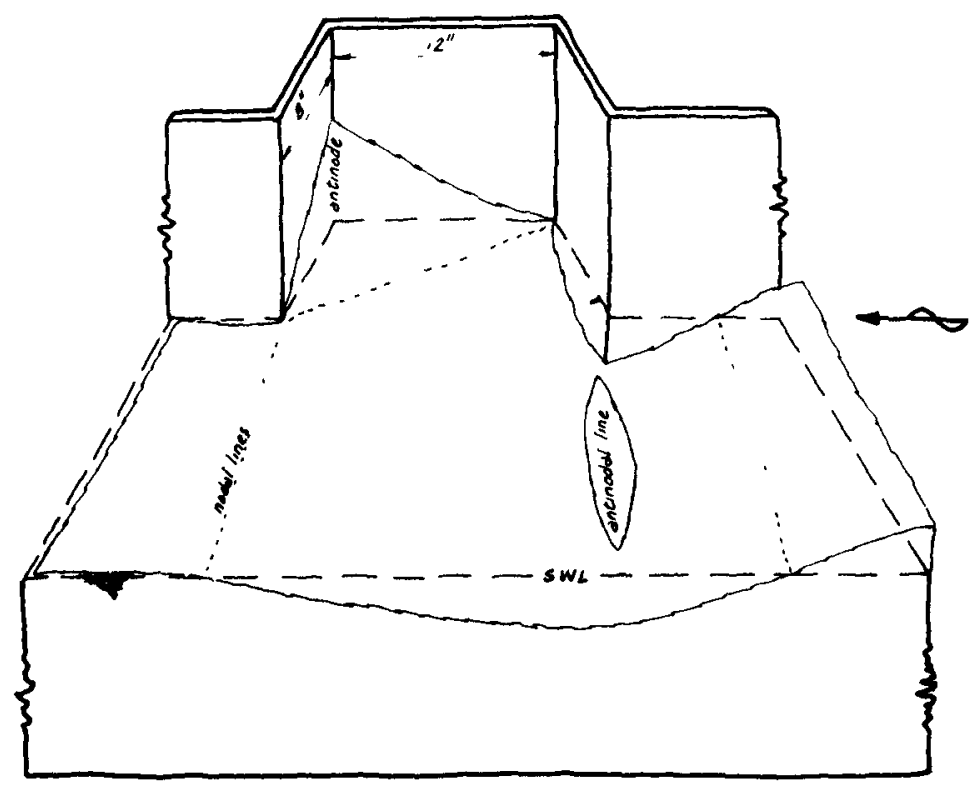

Fig.11. First mode of resonance.

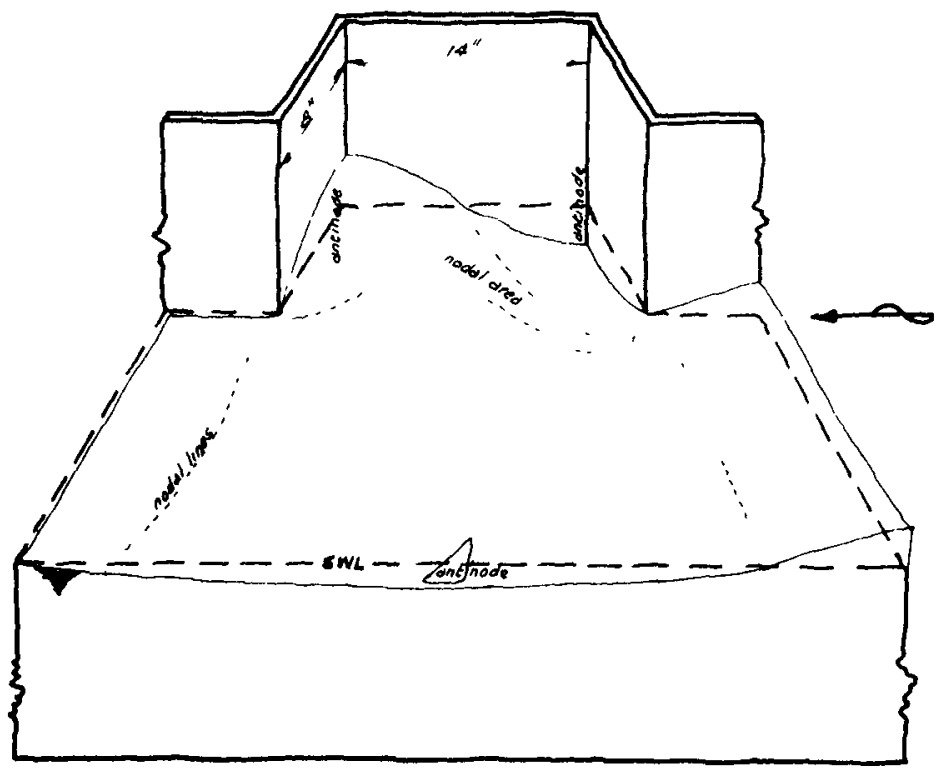

Fig.12. Second mode of resonance. 


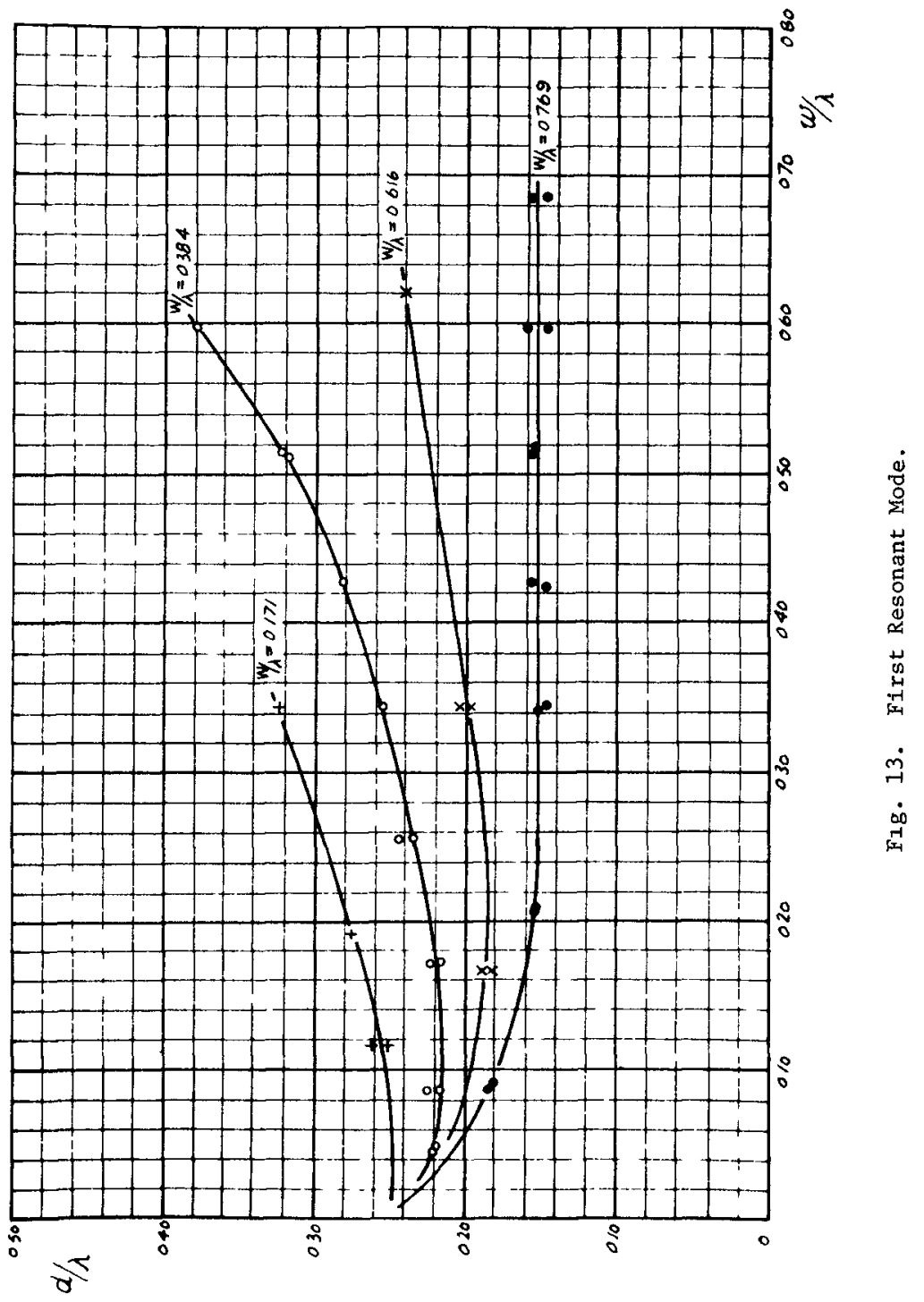


Waves of the characteristic resonance frequency are not transmitted past the resonator but are reflected upstream as negative waves.

3. The zone of protection provided by a resonator is limited to a w1dth of $\lambda / 2$ in the downstream domain. Consequently, the benefits of resonators vanish as $W$ increases beyond $\lambda$.

4. Resonant amplitudes of vertical oscillation in a resonator are finite, irrespectrve of viscous dissipation.

5. The cardinal point established in this investigation, is that resonator geometry for resonance differs greatly from earlier recommendations. of utmost significance is the width of the entrance channel. Harbour entrance geometry at resonance is accurately related to incident wavelengths by the family of curves in Fig.13, which has 1mmediate application in design.

6. The frequency band width for which $\beta$ is less than any prescribed value is a function of $w / \lambda$ (and increases directly with increase $1 \mathrm{n} w / \lambda$ ) as well as a function of $W / \lambda$.

The dependence of the tuning parameter $d / \lambda$ on $w / \lambda$ at resonance is such that a minimum exists at $w / \lambda<0.2$. Generally, however, the tuning parameter increases with increase in $w / \lambda$.

These two points indicate a compromise in the selection of $w$ and $d$.

7. Optimum resonator performance is related to the energy dissipated in the resonator. At resonance, $\alpha=1-\beta$ approximately. Optimum performance appears to be relatively sensitive and further experiments, in which viscosity is carefully controlled, may be useful to $f_{1 x}$ absolute values. (In these tests there was no temperature control and changes of viscosity of up to $20 \%$ were involved.)

8. Finite wave heights reduce the effectiveness of the resonator. The effect appears to be small, however. Wave height has no influence on resonator geometry for resonance.

9. Sma11 values of $w / \lambda$ are accompanied by both decreased efficiency and high resonant velocities. Such velocities could prove a serious navigational hazard.

10. Where there is partial reflection in domain 2 the resonator should be located at $1=n \lambda / 2(n=1,3,5 \ldots)$, or at the position of a loop if reflections are distributed. Ad hoc model tests would then be advisable.

11. Still water depth is of little or no consequence in the resonator geometry for resonance.

12. Non-uniform depths effectively reduce performance. However, the tuning parameter could be substantially reduced in the case of long waves and considerable savings in financial outlay could then accrue.

13. Current techniques used for the reduction of finite partial clapotis incur unreasonable errors, because of the trochoidal waveform. Suitable corrections can be applied easily. Current wave-flume measuring 
techniques also incur systematic errors through viscous attenuation and menisci effects. Corrections for these can be easily applied. The random error encountered in similar investigations need not exceed $1 \%$ if a similar measuring procedure and experimental apparatus is adopted.

\section{ACKNOWLEDGEMENTS}

Guidance from Prof. J. Allen and financial assistance from the S. African C.S.I.R. and the University of Natal is gratefully acknowledged.

\section{NOTATION}

$w$ resonator width

W channel width

$\lambda$ wavelength

$\eta$ surface ordinate

a semi-amplitude

$\bar{\alpha}$ complex vector

|⿰) reflectivity

J $\sqrt{-1}$

$\hat{\alpha}$ phase angle

$k$ wave number

$x$ horizontal abscissae

$\sigma$ circular frequency

$t$ time

$\hat{i}$ unit vector in $x$ direction

$|\beta|$ transmissivity

c a constant

$\psi$ stream function
1 length of an equipotential

$|R|$ transmissivity for resonator

|A| reflectivity in resonator

$y$ vertical ordinate

h still water depth

$\varnothing$ veloc1ty potential

E total energy

$\rho$ mass density

$g$ gravitational acceleration

$z$ transverse co-ordinate

$\mathrm{H}_{\mathrm{L}}$ equivalent head loss

$U$ a generalised velocity

$K$ dimensionless coefficient

$d$ length of resonator

$\bar{q}$ velocity vector

$s$ distance along an equipotential.

Subscripts 1,2 , $R$, refer to semi-infinite ocean, downstream domain and resonators respectively.

Subscripts I, II refer to mode $I$ and mode II respectively.

\section{REFERENCES}

1. Valembois, J. "Etude de 1'Action d'Ouxrages Resonants sur la Propagation de 1a Houle", Proc. Minnesota Int. Hyds. Conf., Minneapolis, 1953, pp 193-200.

2. Penny, W.G., and Price, A.T. "The diffraction theory of sea waves and the shelter afforded by breakwaters", Phi1. Trans. Roy.Soc., series A, Vo1.244, No.882, March 1952, pp 236-253.

3. Le Mehaute, B. "Theory of wave agitation in a harbour", Jour.ASCE, HY9, March 1961, paper 2765, pp 31-50.

4. Hami11, P.A., "Experimental development of a perforated wave absorber of simple construction and minimum length", Mech. Eng. Report MB-252, N.R.C. No.7472, Ottawa, Canada, May 1963. 